\title{
Acknowledgement to reviewers
}

The editorial board wishes to thank the following individuals who have helped to review manuscripts for European Journal of Endocrinology during 2016. Their assistance is gratefully acknowledged.

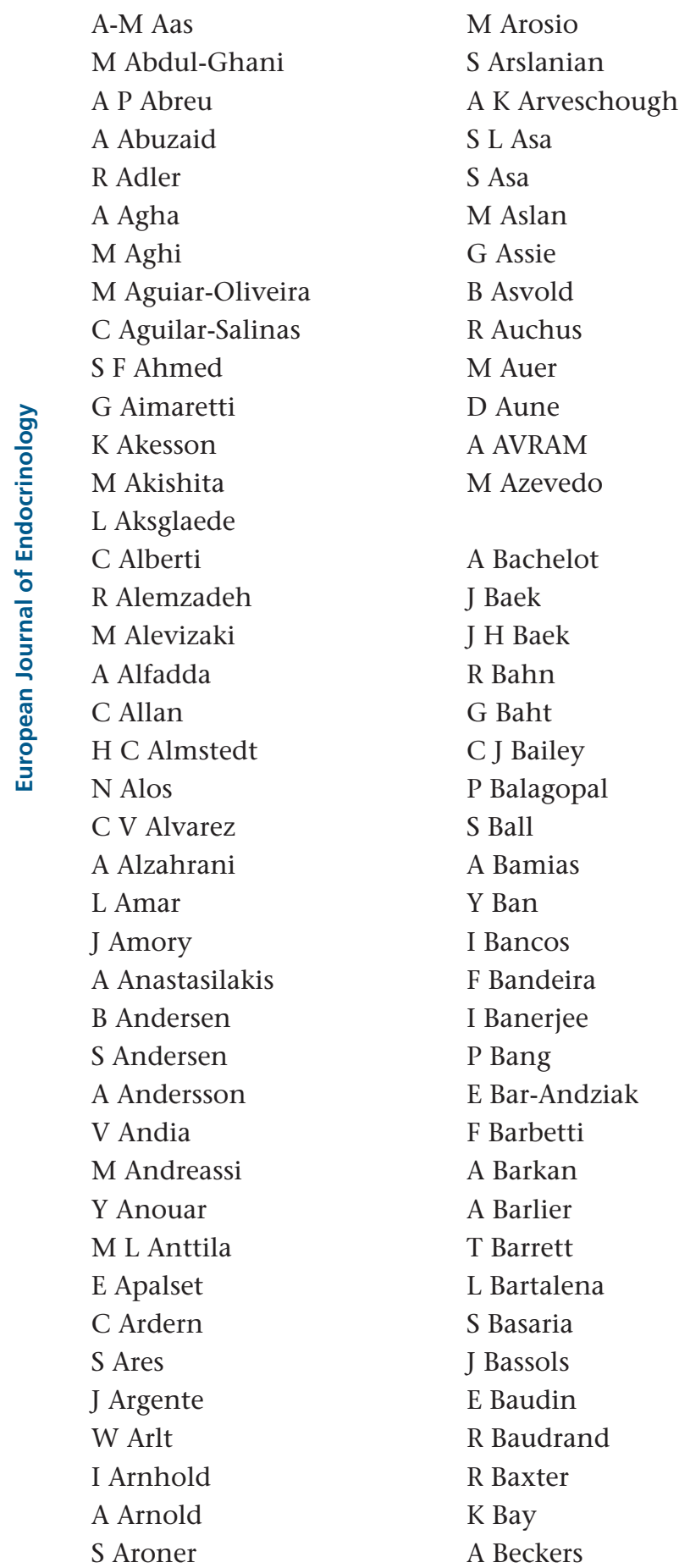

www.eje-online org DOI: 10.1530/EJE-176-A1

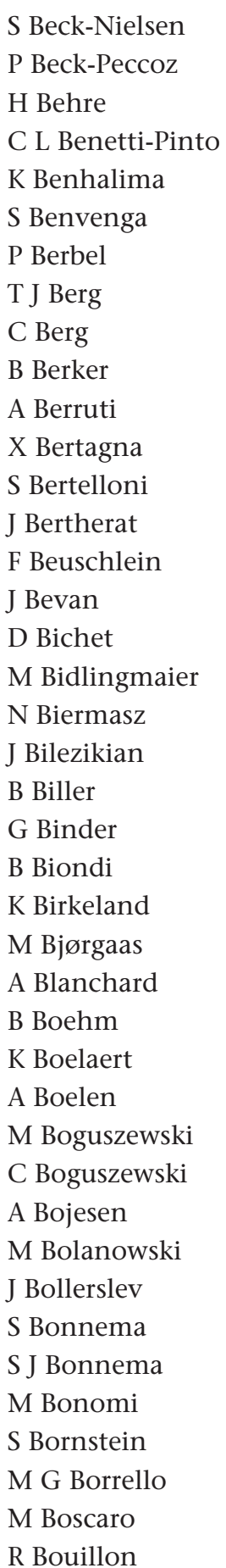

I Bourdeau

A Boyce

M Bozzola

G Brabant

G E Breitwieser

G Brigante

M Bronstein

E Brown

J Brown

M Buchfelder

C Buffet

A Burger

S Burger-Stritt

P Burman

R Buzzetti

G Cadiot

E Cakal

A Campos-Barros

M Cappa

C Cappelli

A Cappola

A Carcavilla

J-C Carel

A Carle

S Carlsen

H Carlson

P Caron

$\mathrm{T}$ Carpenter

A Carrascosa

P Carroll

R Carroll

G Carvalho

D Carvalho

O Casar-Borota

M Castagna

F Castinetti

A Catalano

G Cavallaro

L Cazabat

F Cetani

Published by Bioscientifica Ltd. 


\begin{tabular}{|c|c|c|c|}
\hline T-C Chang & F de Zegher & E Ferrante & A Glezer \\
\hline J P Chanoine & C Deal & S Ferrari & S Goede \\
\hline P Chanson & M Debono & H Filipsson Nyström & B Goichot \\
\hline M Charlton & O Dekkers & E Fischer & C Gomez-Sanchez \\
\hline E Charmandari & L Delbridge & M L Fleiuss de Farias & L Gooren \\
\hline A Chatzigeorgiou & B Delemer & M Fleseriu & P Gorden \\
\hline T Cheetham & P Delhanty & C Foresta & $\mathrm{p}$ gosse \\
\hline W Chemaitilly & E Diamanti-Kandarakis & P Foster & P Goudet \\
\hline F Chiarelli & G Díaz-Soto & F Foufelle & C H Gravholt \\
\hline C Chiesa & J Díez & J Franklyn & Y Greenman \\
\hline I Chiodini & A Diez-Perez & S Franks & V Grill \\
\hline A Chiti & M Donath & F Frasca & A Grossman \\
\hline S H Choi & H Doneray & A Frasoldati & L Guignat \\
\hline C Choong & H Dralle & A Frederiksen & D Gullo \\
\hline J A Chowen & M Drent & K Froeslie & T Gurlo \\
\hline E Christ & R Dullaart & L Frohman & M Gurnell \\
\hline G Chrousos & D Dunger & M Frost & \\
\hline A Cignarelli & L Dunkel & L Fugazzola & S Haddad \\
\hline K Claessen & W Dunn & D Führer-Sakel & S Hahner \\
\hline B Clarke & F Dunne & Y Fujisawa & M Haluzik \\
\hline P Clayton & L Duntas & H Fukuoka & N Hamdy \\
\hline D Clemmons & C Durante & J Funder & M Hammami \\
\hline J Clerc & E Duregon & J Fuqua & P Hammel \\
\hline A Clerico & A Dwyer & & A Hamrahian \\
\hline A Colao & & M Gadelha & D Handelsman \\
\hline P Colman & K Eckardt & J Gallagher & J Hangaard \\
\hline C Colón & Y Eisenberg & I Gallos & D Hanley \\
\hline F Conceicao & G Eisenhofer & J Galofré & F M Hannan \\
\hline F Conti & B Ekman & A Gambineri & R Haring \\
\hline A Coomarasamy & R Elisei & M A Ganie & A O Hasdemir \\
\hline M Cooper & C Ellervik & M Garabedian & J Hazlehurst \\
\hline J-B Corcuff & M Erdogan & R Gärtner & A Heck \\
\hline M Courey & C Erem & B Gatta-Cherifi & G Hendy \\
\hline \multirow[t]{2}{*}{ R Coutant } & M Eren & E Gault & A Hermus \\
\hline & E M Erfurth & B Gaylinn & T Hernandez \\
\hline M d'Herbomez & E Eugster & L Gennari & M Hero \\
\hline P Dahia & S Ezzat & E Ghigo & B Herrmann \\
\hline J Dahlgren & & D Gianfrilli & H Heuer \\
\hline P Damm & G Fadda & L Gianotti & C Higham \\
\hline M Datani & A Faggiano & C Gibbons & P Hindmarsh \\
\hline M Dattani & A Faje & J Gibney & O Hiort \\
\hline C Daumerie & F Faletra & M Gillman & Y Hirata \\
\hline B Dawson-Hughes & H Falhammar & E Giltay & D Hirsch \\
\hline C Dayan & A Falorni & A Gimenez-Roqueplo & K Ho \\
\hline W de Herder & M Fassnacht & A-P Gimenez-Roqueplo & L Hofbauer \\
\hline E de Koning & P Fazeli & J Ginsberg & J Hoffstedt \\
\hline R de Krijger & R Feelders & R Giordano & P Hofman \\
\hline N De Roux & U Feldt-Rasmussen & C Giordano & D Hofsø \\
\hline J De Schepper & B Feldt-Rasmussen & 1 giovanella & K Højlund \\
\hline S De Sousa & J-M Fernandez-Real & A Giustina & A Hokken-Koelega \\
\hline
\end{tabular}




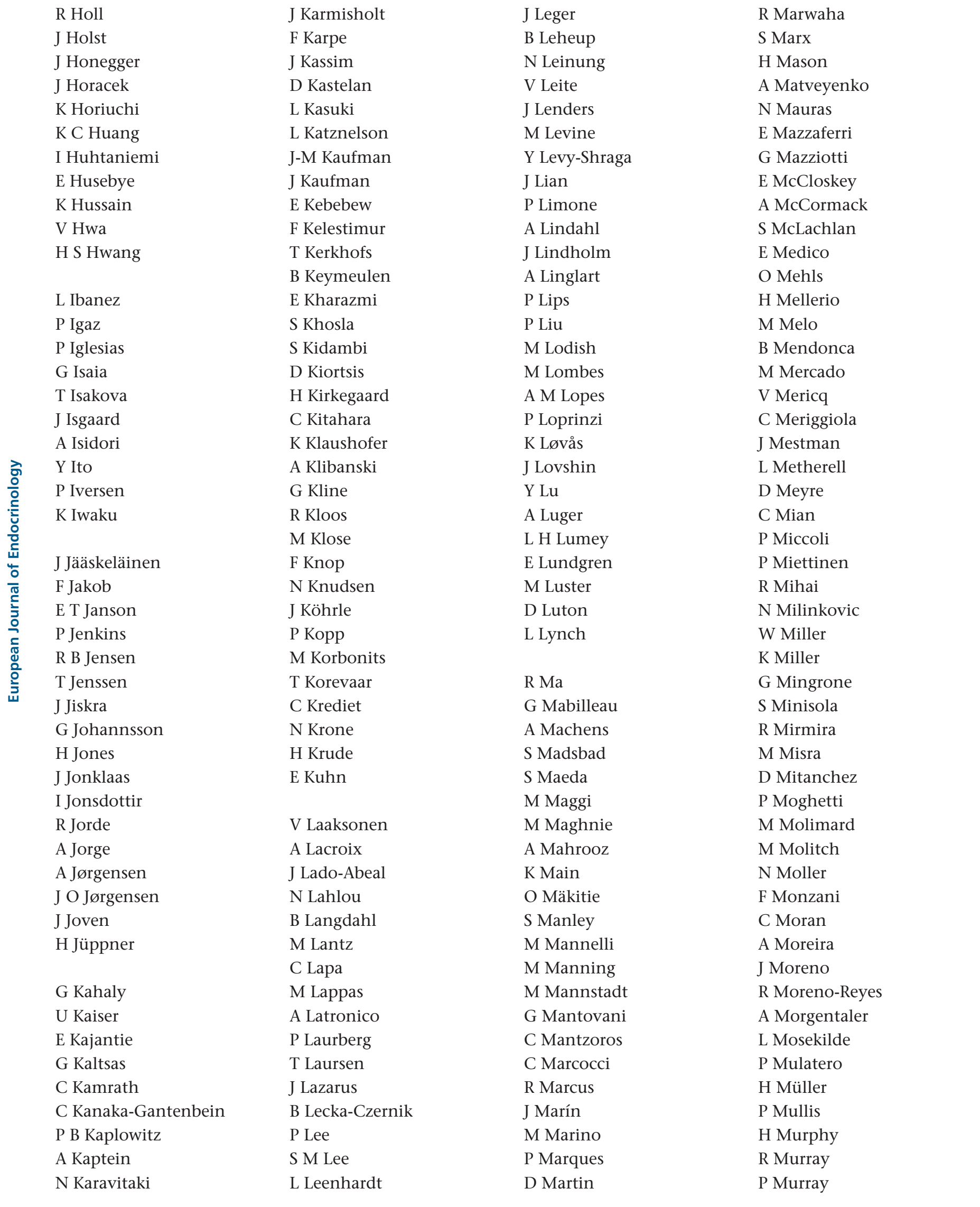




\begin{tabular}{|c|c|c|c|}
\hline M Muzza & R Pasqualli & G Reimondo & C Schöfl \\
\hline & G Paz Filho & M Reincke & U Scholl \\
\hline Y Nakano & M Peacock & T Reinehr & J Schopohl \\
\hline N Napoli & E Pearce & N Reisch & P Schrauwen \\
\hline S Narumi & S Pearce & L Rejnmark & F Schreiner \\
\hline S Neggers & J Pecina & J Renes & J Schwartz \\
\hline R Negro & F Pecori Giraldi & Y Reznik & A Sejling \\
\hline N Negro & R Peeters & A Riester & A-S Sejling \\
\hline I Netchine & A Pereira & P Riva & S Seminara \\
\hline H Neumann & A Peri & R Rizzoli & J Sgarbi \\
\hline M New & P Perros & N Rodondi & A M Shah \\
\hline K Newbold & L Persani & G Roef & S Shalet \\
\hline J Newell-Price & S Petersenn & F Roelfsema & C Shaw \\
\hline M Niedziela & L A Phillips & A Rogol & M Sherlock \\
\hline S Nielsen & S Pianna & L Rolighed & M Sherlock \\
\hline L Nieman & A Piccardo & C Romei & I Shimon \\
\hline E Nieschlag & A Pico & J Romijn & C S Shin \\
\hline P Nightingale & F Pitoia & C Ronchi & A Shinkov \\
\hline H Nishioka & R Pivonello & C Ronin & D Shoback \\
\hline A Nordenstrom & M Polak & R Rosenfeld & 1 sidossis \\
\hline N Nota & M Pombo & M Rosilio & T Sikjaer \\
\hline G Ntali & V Popović & J Ross & C Silve \\
\hline V Nunes & P Poulsen & I Ross & S Silverberg \\
\hline & C Powe & R Ross & M Simmonds \\
\hline S Oberfield & C Pozza & G Rossi & D Simmons \\
\hline K Oberg & P Pozzilli & L Rostomyan & N Simon \\
\hline M Obregon & C Pretorius & C Roth & F Singer \\
\hline R Ogrin & & M Rotondi & N Skakkebaek \\
\hline C Oktenli & D Quarles & J Rubin & J Skupien \\
\hline D Olsson & M Quinkler & M Ruchala & J Smit \\
\hline T Olsson & R Quinton & G Russ & J smyczynska \\
\hline K Ong & E Qvigstad & & P Soares \\
\hline M O’Reilly & & W Saeger & O Soder \\
\hline J Orly & S Radovick & M Saito & S J Soldin \\
\hline A Osswald & A Rafacho & A Sakamoto & O Soldin \\
\hline E O'Sullivan & H Raff & E Sala & M Solter \\
\hline V Ott & O Ragnarsson & M Salerno & C Sommer \\
\hline B Otten & T Raivio & R Salvatori & I Song \\
\hline M J Oursler & J Rajpura & M Salvi & I-S Song \\
\hline J Overbeek & H Ramos & M H Samuels & P Sönksen \\
\hline M Owecki & M Ranke & R Sánchez-Borrego & P Spritzer \\
\hline K Ozono & D S Rao & A Sansone & A Stagnaro-Green \\
\hline & R Rapaport & P Santisteban & T Stalder \\
\hline K Pacak & G Rappold & M Santoro & G Stalla \\
\hline F Pacini & F Rauch & A Santos & M N Stan \\
\hline F Pagni & F Raue & M Satoh & L Stene \\
\hline E Papini & G Raverot & M Savage & C Stettler \\
\hline K Parhofer & S Razvi & S Sbiera & A Stewart \\
\hline Y J Park & D A Rees & C Schmid & R Stimson \\
\hline R Pasquali & S Reffetof & H Schneider & D Stocco \\
\hline
\end{tabular}




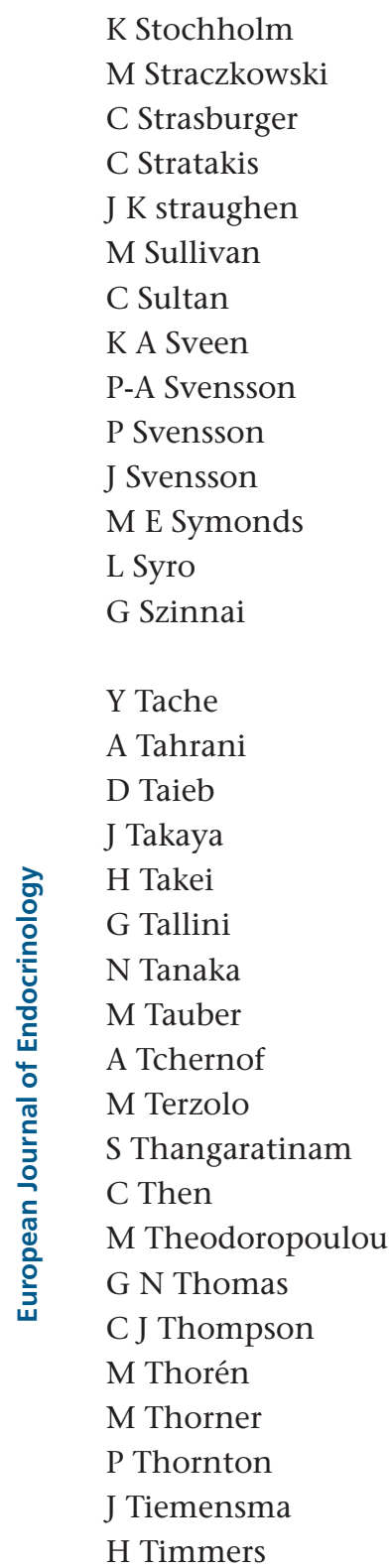

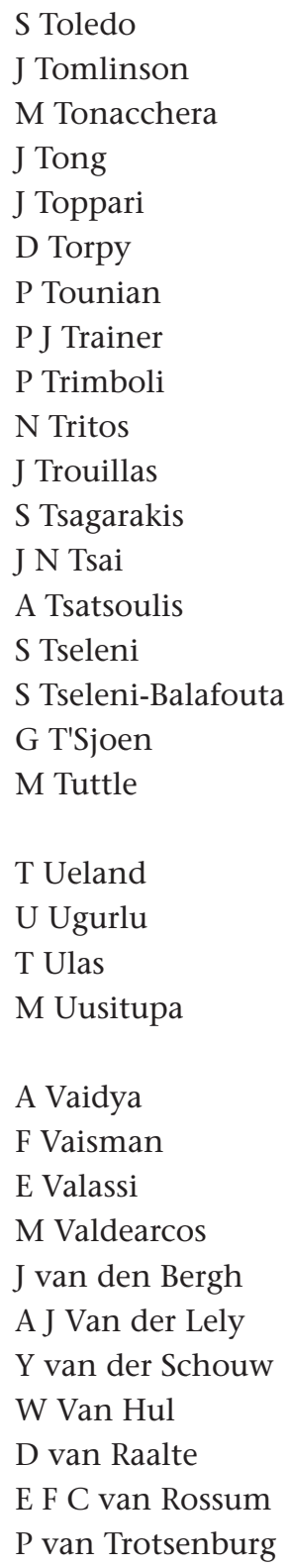

\begin{tabular}{|c|c|}
\hline M Vanderpump & D Weismann \\
\hline K Varady & S Wells \\
\hline J Vassallo & J-L Wemeau \\
\hline J Vega & D Werny \\
\hline I Velasco & B Wheeler \\
\hline L Velloso & M Whitaker \\
\hline F Verburg & W Wiersinga \\
\hline P Vestergaard & L E Wildemberg \\
\hline D Vezzosi & J Wit \\
\hline F Vianello & B Wolffenbuttel \\
\hline J G Vieira & Y-W Wu \\
\hline 1 vignozzi & $\mathrm{F} \mathrm{Wu}$ \\
\hline \multicolumn{2}{|l|}{ L Vilar } \\
\hline T Vilsbøll & M Xing \\
\hline \multicolumn{2}{|l|}{ A Vinik } \\
\hline A Virdis & S Yamada \\
\hline R Vita & P Yang \\
\hline M Vitale & J A Yanovski \\
\hline P Vitti & C Yates \\
\hline M Volante & B Yeap \\
\hline H Volzke & M Yilmaz \\
\hline O Vonend & H Yokokawa \\
\hline S B Votruba & J Young \\
\hline \multirow[t]{2}{*}{ R Voutilainen } & W Young \\
\hline & G Yuan \\
\hline M Wabitsch & K Yuen \\
\hline \multicolumn{2}{|l|}{ A Wagenmakers } \\
\hline H Wagner & S Zanotti \\
\hline B Walker & M C Zatelli \\
\hline J Wass & Z Zhou \\
\hline T Watt & M Zitzmann \\
\hline S Webb & T Zueger \\
\hline \multicolumn{2}{|l|}{ G Weber } \\
\hline \multicolumn{2}{|l|}{ L Weinstein } \\
\hline L S Weinstein & \\
\hline
\end{tabular}

